\title{
Cardiovascular Disease Patient Prevention Experience with Nurses in the Primary Care Sector
}

\author{
Moath Mohamed Farasani* \\ Prince Mohamed Bin Nasser Specialist Hospital, Saudi Arabia
}

\begin{abstract}
Introduction: Cardiovascular disease (CVD) is a common term used to refer to disorders affecting the blood vessels or the heart that have led to the majority of deaths worldwide.
\end{abstract}

Aim: The aim of the present study was to explore nurse's perception of their role and their experiences in relation to engaging with cardiovascular disease prevention in the primary care setting.

Methods: In the selection of clinical nurses who were interested in primary care to participate, qualitative describing design and impracticable sampling were used. The data collection methods include semi-structured and one-on-one interviews. The results were analyzed by the methodology for qualitative content analysis.

Results: In this study, 10 nurses were participated and their role in CVD prevention plays a part in health education, diagnosis and consultation, reassurance, monitoring of vital signs, and collecting blood samples. The challenges include cultural obstacles and vocabulary, negative attitudes, contradictory regulations, a lack of equipment, a shortage of nursing staff and the lack of knowledge and information. Electronic information, patient education, nurse training, and communication workshops are the facilitators for nurses that prevent heart disease.

Conclusion: Cardiovascular disease is lethal, but adequate preventive programs can accommodate it. In addition, nurses play an important role in preventing CVD in primary healthcare and should therefore concentrate efforts on the enhancement of their capacity. The aim of future research should be to understand how patients interpret and incorporate data on the prevention of CVD provided by nurses.

Keywords: Cardiovascular disease, Coronary artery disease, Stroke, Nurses, Primary care

\section{Introduction}

The world's leading threat continue to be cardiovascular diseases (CVD) such as coronary heart disease, pulmonary respiratory disease, heart failure, stroke, rheumatic heart disease and hypertension. Approximately all CVDs inevitably lead to heart failure, which is a serious disease with significant morbidity and mortality and a major burden not just for the affected persons but also for community and health care systems. ${ }^{1}$ According to the Centers for Control and Prevention, most chronic diseases such as CVDs, stroke, obesity and diabetes are preventable and less costly to treat wheat and are known to account for over $44 \%$ of deaths. ${ }^{2,3}$ The pathogenesis of
CVD is dominated by atherosclerosis. ${ }^{4}$ The risk factors of activities such as obesity, smoking, unhealthy diet, alcoholism and physical inactivity will prevent the CVD. Due to the fact that the disease is the leading cause of death, it is important to assess the role of nurses in prevention, as early detection helps start suitable interventions which reduce mortality. ${ }^{5}$ Nurses play an important role in cardiovascular disease prevention and treatment, a strategy that aims to minimize people's heart disease. In particular because of the need to integrate health education and wellness promotional activity in their positions, ${ }^{6}$ nurses play an important role in preventing CVD in China based on Ma and Zhu's quantitative survey. The role of
Quick Response Code:

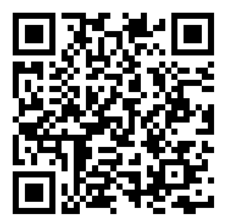

\author{
*Corresponding author: Moath Mohamed Farasani, Senior Specialist Nurse, Prince Mohamed \\ Bin Nasser Specialist Hospital, Jazan-82511, Saudi Arabia \\ Received: 16 January, 2021 \\ Published: 03 February, 2021 \\ Citation: Moath Mohamed Farasani. Cardiovascular Disease Patient Prevention Experience \\ with Nurses in the Primary Care Sector. SOJ Complement Emerg Med. 2021;1(1):1-5. DOI: \\ 10.53902/SOJCEM.2021.01.000501
}


nurses in the prevention of CVD is divided into three categories: primary prevention, clinical procedures, and disease management. Around $12 \%$ of nurses in Switzerland create plans to avert risky habits and risk factors contributing to heart disease. ${ }^{7}$ This is in line with the Tagliabue et al survey conducted in Switzerland. The research also shows that the caregivers provide guidance, health education and review of previous CVDs. Tagliabue et al. further claimed that nurses provide outreach programs to encourage good health and heart disease prevention. Interventions used by nurses to avoid CVD apply to the efforts of nurses to tackle CVD. According to the longitudinal, pre-test-posttest, pre-test community design performed in Mexico and Costa Rica between 2011 and 2012, nursing operations include patient training, therapy, evaluation, and management of outreach services in cardiac disease prevention. ${ }^{8} \mathrm{Global}$ wide studies have been shown the role of nurses and their experience in engaging the prevention of CVD in the primary cares and this study shows the role of nurses from the Saudi Arabia.The aim of the present study was to explore nurse's perception of their role and their experiences in relation to engaging with cardiovascular disease prevention in the primary care setting.

\section{Materials and Methods}

\section{Research methodology}

The inclusion criteria for this study are all registered nurses working in primary care in direct patient contact roles and the exclusion criteria are nurses who are involved in administrative roles in primary care, student nurses and assistant nurses. The study will take place in a comfortable room within the primary care centers. The main approaches used for research are quantitative and qualitative. Quantitative research is a study involving methods that stress objective measurement, mathematical, statistical and numerical analysis of the information collected. Quantitative research is based on the philosophic perspective of the positivist paradigm, while qualitative research is a nominal data collection that seeks to understand the phenomenon based on the philosophic perspective of phenomenology. In comparison, qualitative study data are analyzed by themes for descriptions while quantitative study data are analyzed by means of statistical inferences and numerical comparisons. In addition, the qualitative study assumes a negotiated and dynamic reality, while the quantitative study assumes a measurable and fixed reality. Qualitative methodology was used in this study because it involves understanding human behavior by collecting data through interviews. Major qualitative approaches are ethnography, grounded theory and phenomenology. Ethnography focuses on the population's traditions through the participant observation approach as part of the field study. Grounded theory focuses on raising generative questions that help guide the study but are not intended to be either confined or static. Phenomenology is a collection of thoughts that focus on people's subjective interpretations and experiences of the world in order to understand how the world appears to others. The qualitative descriptive approach is a scientific method that involves observing and describing the characteristics of the subject, but does not in any way influence it. Qualitative descriptive design is based on a pragmatic paradigm. In addition, the presentation of data in the qualitative descriptive study consists of a summary of information organized in a logical sequence. ${ }^{9,10}$ However, the data in the descriptive study is structured according to the different techniques used to obtain the data. The main justification for a qualitative descriptive design is to describe, explain and validate data, whereas the description follows creative research with the objective of manipulating the information in accordance with discussion and explanations. ${ }^{11}$ The main benefits of qualitative design are that they are not manipulated by the subject of the study. Furthermore, the topics and issues covered are thoroughly assessed as the interviews are not limited to specific questions. When the new findings and information emerge, the framework and direction of the study can be quickly revised. Furthermore, it depends on human experience to be compelling and powerful the information collected in qualitative descriptive design. The downside of qualitative descriptive design is that the collected data must be coded in order to carry out an analysis so that a miscoding is not possible..$^{10,11}$

\section{Sample and setting}

In the southern part of Saudi Arabia, nurses will represent the population of interest. Two samples are available: probability and unlikely. Probability sampling is the sampling technique that uses some form of random selection using the collection of procedures to ensure that the same opportunities for selection are open to individual units in the sample population. Sampling is not probability, and thus the samples does not depend on the reasoning for probability theory. The sampling process does not require probability and random selection samples. The type of sample used in this trial is not likely to be sampled on the basis of population characteristics and research objectives. Five different types of techniques for unlikely sampling exist: quota, ease, careful selection and snowball. The method of sampling used in the analysis is to make a targeted sample. It is particularly useful in qualitative research as it is definitive when explaining the phenomenon of interest. The reason for the use of purposeful sampling is that This method of sampling was used to select clinical health workers at primary care centers in Saudi Arabia's south-west region. However, because of the wealth of knowledge obtained from participants and the great number of verbal information to be evaluated, the sample size is limited. A priori, it is estimated that the sample size would be around 12 nurses. When the gathering of new knowledge does not shed further light on the issue under consideration, the sample size would follow the principle of saturation..$^{9,12}$

\section{Data analysis}

Qualitative content analysis was used to analyse the data. The reason for using qualitative content is because it makes use of subjective judgment depending on information that is non-quantified such as research and development. There are two different kinds of qualitative content analysis which include manifest and latent. ${ }^{10}$ However, this study used latent analysis come up with different categories and subcategories of the qualitative data that will be obtained..$^{10}$ The data was transformed from audio tape into paper by 
listening to the tape in a silent room after and then documenting the information. The qualitative content that is phrases/sentences

Table 1: Role of nurses in categories and sub-categories of services.

\begin{tabular}{|c|c|c|}
\hline Theme & Categories & Subcategories \\
\hline \multirow[t]{2}{*}{ Nurses role } & Health Education & $\begin{array}{c}\text { Causes Risks } \\
\text { Prevention - diet, exercise, }\end{array}$ \\
\hline & Assessment & Vital signs, Investigations (ECG, blood sugar, cardiac enzymes) \\
\hline \multirow[b]{2}{*}{ Barrier } & Top barriers & Lack of facilities and unclear policy \\
\hline & Difficulties & $\begin{array}{l}\text { Un updated policies Nurse shortage Design of the facility Language barrier Inadequatenutritionist } \\
\text { Lack of knowledge and information source } \\
\text { Cultural barriers Patient attitudes }\end{array}$ \\
\hline \multirow{3}{*}{ Facilitators } & Patient health education & Creating awareness \\
\hline & Nurse training & Lectures and workshop Improving nurses knowledge \\
\hline & Proper investigation & Assessment \\
\hline
\end{tabular}

\section{Results}

Table 2 provides the demographic details of the participants. It took 45 years for the oldest nurse to participate while 20 years for the smallest. The mean age was 33.5 years, however. Most nurses were over 40 years of age. Most of those interviewed were women $(n=7)$. The Table 2 also demonstrates how nurses were interviewed to the highest degree of education. Nine students had a nursing diploma, with only one getting a baccalaureate degree. The data reveals that a degree from a recognized university only included a few nurses employed in primary care. The average period of experience is 8.2 years, based on the results. But the longest working time was 22 years and the lowest 1 year. The data indicate that nurses with various job backgrounds engage in the health sector in Saudi Arabia. In addition, for the past three years, $60 \%$ of nurses have been attending cardiovascular workshops while $40 \%$ do not. The infor-

Table 2: Demographic data of the participants.

\begin{tabular}{|c|c|}
\hline Demographic Information & $(\mathbf{N}=\mathbf{1 0})$ \\
\hline Age & $3(3 \%)$ \\
\hline $20-29$ & $5(5 \%)$ \\
\hline $30-39$ & $2(2 \%)$ \\
\hline $40-49$ & $3(3 \%)$ \\
\hline Gender & $7(7 \%)$ \\
\hline Male Female & $1(1 \%)$ \\
\hline Level of education & $9(9 \%)$ \\
\hline Bachelor Diploma & $8(8 \%)$ \\
\hline & $1(1 \%)$ \\
\hline Years of clinical experience in primary care & $1(1 \%)$ \\
\hline $0-9$ & \\
\hline $10-19$ & $6(6 \%)$ \\
\hline $20-29$ & $4(4 \%)$ \\
\hline Yes & \\
\hline No & \\
\hline
\end{tabular}

was condensed into a meaning unit, coded, grouped into subcategories and later into categories which are presented in Table 1. mation reveals that 6 in 10 primary care nurses still have updates on heart disease treatment. Table 1 offers statistics about how many nurses conduct various activities in primary care, including measuring, blood pressure control, appraisal, reassurance, therapy and education. A primary care nurse with 18years' experience said "I usually check and write the patient's name, blood pressure, weight, and if the patient has diabetes then he can go to his doctor." I normally use his blood glucose file. In addition, nurses are critical roles and interventions such as identifying vital signs of heart disease, diet counseling, stress management and educating patients on risk factors. A nurse with 5 years' experience noted that "their main goal is handling patients with utmost care, taking into considerations of his or her family medical history before proceeding with the diagnosis". According to one of the respondents, nurses in the primary care are required to carry out physical examinations of clients and test blood glucose as well as calculating body mass index. ${ }^{13}$ 


\section{Discussion}

This subject includes factors that improve CVD prevention interventions. The main facilitators of heart disease prevention include ambivalence in the quest for accurate knowledge, lifestyle measures, improvements in physical activity and health education based on Canadian comprehensive cross-sectional surveys ( $n=144$ participants). In addition, treatments that improve effectiveness and readiness for motivational purposes are necessary to avoid and postpone CVD complications in patients with diabetic diseases. ${ }^{14}$ Ambivalence refers to an attitude and a thought conflict. Patient ambivalence provides an incentive for finding more knowledge about CVD, as well as for nurse advice. In encouraging patients to apply lifestyle changes, nurses use behaviors or ambivalence. Initially, ambivalence is the key driving force behind improvements in lifestyle prevention, including CVD. Kehler et al. ${ }^{15}$ said there are various kinds of ambivalence in the qualitative interview in Denmark $(n=12)$, which includes details, care, demand, therapy and perception. Patient knowledge of CVD's danger due to specific dietary habits or behavior is the feeling and contemplation associated with the patients' comprehension. Ambivalence of knowledge is the confusion about the patient's specifics when consulting CVD preventive steps. The quantitative survey conducted by Hardcastle et al in the USA ( $n=107)$ also indicates that ambivalence in therapy relates to the reflexion and sensation of the need for a lifestyle change and medication to alleviate symptoms and compensate for illness. ${ }^{16}$ The current uncertainty about multiple means of preventing heart disease motivates the health care providers to obtain proper and reliable information on effective cardiac disease education. According to the quality focus group of McKillop et al. ${ }^{17}(\mathrm{~N}=20)$, interventions in New Zealand help patients change traits which pose a risk to cardiac disease growth, such as alcoholism, unhealthy diet, and smoking. Lifestyle interventions help to reduce the risk. Similarly, the 2015 U.S. intervention study conducted by Kandula $(n=63)$ showed that interventions of cultural significance and lifestyles in the prevention of cardiovascular disease could engage immigrants in South Asia. ${ }^{18}$ Telephone assistance, behavioral modifications, group courses, and experiential experiences were included. Interventions In addition, the research showed that the prevention of heart disease is promoted through clarification and translation of local language instruction, weight, CVD and weight control messages in education materials.

According to a qualitative analysis by Mattei et al. in the United States ( $=24)$, the prevention of CVD is assisted by health education, healthcare and therapy in health and via social media. ${ }^{19}$ On the basis of a qualitative study conducted in Kandula $(n=36)$, patients are helped to improve actions, such as smoking, unhealthy diet and excessive consumption of alcohol. Patient therapy also encompasses multiple aspects that facilitate good health between patients and the community as different caregivers, such as nutritionists, nurses, physiotherapists and physicians, may provide counseling. ${ }^{18}$ The results of Kandula showed that nutrition education is one of the strategies promoting cardiac disease prevention. The process includes presenting details related to proper dietary intake, changes in lifestyle and how to fulfill balanced dietary requirements by local foodstuffs. The community is trained in the practice of healthy diets through social media and nutrition education incorporation into the curriculum of schools. Summarizing, behavioral improvements, health education, ambivalence and drug strategies are the key facilities for nurses in CVD prevention. Furthermore, CVD preventive interventions are improved by treating other lifestyle disorders, such as diabetes and weight loss services. The strength of this study was to document as 10 nurses from Saudi Arabia working in primary care were enrolled, thus they provided more detailed information and limitation of this study was only 10 nurses have been involved which cannot be generalized to whole Saudi Arabia community.

\section{Conclusion}

To conclude, CVD is the largest worldwide cause of death, with over 17.5 million people suffering from cardiovascular disease. In prevention of CVD, nurses play an important role through the provision of useful information of their continued treatment to patients, including education, therapy and evaluation and through leading outreach initiatives. Through healthcare, nurses will alter unhealthy characteristics and lifestyles, which predispose you to heart disease. This study concludes as nurses play a major contributing role and apply their experience in dealing smartly with the patients diagnosed with CAD in the primary care setting. This study confirms an overview of CAD by presenting information on cardiovascular diseases and primary care management to support the methodological aspect.

\section{Acknowledgments}

None.

\section{Statement of Ethics}

None of the patients' samples or animal subjects were involved in this study.

\section{Conflicts of interest}

None.

\section{Funding}

None.

\section{Author Contribution}

MMF is the single author, completely responsible for preparation of this manuscript.

\section{References}

1. Chen XF, X Chen, X Tang. Short-chain fatty acid, acylation and cardiovascular diseases. Clinical Science. 2020;134(6):657-676.

2. Musonge-Effoe JE. The association between health care coverage and prevalence of cardiovascular diseases and diabetes over a 10-year period. Preventive Medicine. 2020;132:105983.

3. Khan IA. Correlation between KCNQ1 and KCNJ11 gene polymorphisms and type 2 and post-transplant diabetes mellitus in the Asian Indian population. Genes Dis. 2015;2(3):276-282.

4. Matam K. Coronary artery disease and the frequencies of MTHFR and PON1 gene polymorphism studies in a varied population of Hyderabad, Telangana region in south India. Journal of King Saud University-Science. 2015;27(2):143-150. 
5. Booth FW, CK Roberts, MJ Laye. Lack of exercise is a major cause of chronic diseases. Comprehensive Physiology. 2011;2(2):1143-1211.

6. MaX, S Zhu. Metabolic syndrome in the prevention of cardiovascular diseases and diabetes-still a matter of debate? Europeanjournal of clinical nutrition. 2013;67(5):518-521.

7. Tagliabue L. Aspirin for the primary prevention of cardiovascular diseases. Internal and emergency medicine. 2012;7(4):375-379.

8. Fort MP. Impact evaluation of a healthy lifestyle intervention to reduce cardiovascular disease risk in health centers in San José, Costa Rica and Chiapas, Mexico. BMC Health Services Research. 2015;15(1):577.

9. Creswell JW. A concise introduction to mixed methods research. SAGE publications. 2014

10. Sandelowski M. What's in a name? Qualitative description revisited. Research in nursing \& health. 2010;33(1):77-84.

11. Graneheim UH, B Lundman. Qualitative content analysis in nursing research: concepts, procedures and measures to achieve trustworthiness. Nurse education today. 2004;24(2):105-112.

12. Muna WF. Comprehensive strategies for the prevention and control of diabetes and cardiovascular diseases in Africa: future directions. Progress in cardiovascular diseases. 2013;56(3):363-366.

13. Khan IA. Genetic confirmation of T2DM meta-analysis variants studied in gestational diabetes mellitus in an Indian population. Diabetes MetabSyndr. 2019;13(1):688-694.
14. Grace SL. Physical activity behavior, motivational readiness and self-efficacy among Ontarians with cardiovascular disease and diabetes. Journal of Behavioral Medicine. 2007;30(1):21-29.

15. Kehler D. Ambivalence related to potential lifestyle changes following preventive cardiovascular consultations in general practice: a qualitative study. BMC Family Practice. 2008;9(1):50.

16. Hardcastle SJ. Effectiveness of a motivational interviewing intervention on weight loss, physical activity and cardiovascular disease risk factors: a randomised controlled trial with a 12-month post-intervention follow-up. International journal ofbehavioral nutrition and physical activity. 2013;10(1):40.

17. McKillop A, J Crisp, K Walsh. Barriers and Enablers to Implementation of a New Zealand-Wide Guideline for Assessment and Management of Cardiovascular Risk in Primary Health Care: A Template Analysis. Worldviews on Evidence-Based Nursing. 2012;9(3):159-171.

18. Kandula NR. Translating a heart disease lifestyle intervention into the community: the South Asian Heart Lifestyle Intervention (SAHELI) study; a randomized control trial. v. 2015;15(1):1064.

19. Mattei J. Perceptions and motivations to prevent heart disease among Puerto Ricans. American journal of healthbehaviour. 2016;40(3):322-331. 